\title{
Zur Kenntnis des Verlaufs der fermentativen Polypeptidspaltung.
}

\author{
VI. Mitteilung. ${ }^{\text {) }}$ \\ Von
}

Emil Abderhalden und Carl Brahm.

(Aus dem physiologischen Institute der tierärztlichen Hochschule, Berlin.)

(Der Redaktion zugegangen am 26. August 1908.)

Wir haben uns die Aufgabe gestellt, die Frage zu entscheiden, ob die in den verschiedensten Geweben des Tier- und Pflanzenreiches vorhandenen peptolytischen Fermente identisch sind oder aber eine ganz verschiedene Wirkung zeigen. Dieses Problem mußte sich auf Grund der bisherigen Beobachtungen über den Abbau synthetischer, optisch-aktiver Polypeptide lösen lassen. Unser Versuchsplan war folgender. Die peptolytischen Fermente verschiedener Herkuuft spalten bestimmte Dipeptide verschieden rasch. Es beruht dies unzweifelhaft zum größten Teil darauf, daß sie in den verschiedenartigen Preßsäften und Sekreten in verschiedener Konzentration vorhanden sind. Wir verglichen z. B. Darmsaft und Hefepreßsaft und verwendeten d-Alanyl-glycin. Dieses Dipeptid wurde, wie die folgende Tabelle zeigt, vom Hefepreßsaft in kürzerer Zeit gespalten, als vom Darmsaft (Versuch 1 u. 2, S. 343).

Wir stellten nun den Hefepreßsaft in der Weise auf den Darmsaft ein, daß wir ihn solange mit Wasser verdünnten, bis er das genannte Dipeptid in annähernd gleich rascher Weise spaltete, wie der unverdünnte Darmsaft. Wir können so eine bestimmte Fermentlösung als Standardlösung bezeichnen und alle anderen auf sie beziehen. Da wir vorläufig keine Methoden besitzen, um ohne irgend welche Beschädigung von Fermenten Lösungen von solchen einzuengen, so werden wir stets die am schwächsten wirkende Fermentlösung als Grundlage wählen.

1) Vgl. 5. Mitteilung von Emil Abderhalden und A. H. Koelker, Diese Zeitschrift, Bd. LV, S. 416, 1908. 
Über den Verlauf der fermentativen Polypeptidspaltung. VI. 343

Versuch 1.

1,0 ccm d-Alanyl-glycinlösung

0,5 , Hefepreßsaft.

(1/1000-Mol.).

5,0 , Wasser.

Zeit Abgelesener Winkel

0 Minuten $\left.1,00^{\circ}\left(1,07^{\circ}\right)^{1}\right)$

$15 \rightarrow 0,75^{\circ}\left(0,82^{\circ}\right)$

$30 \gg 0,60^{\circ}\left(0,67^{\circ}\right)$

$45 \gg 0,60^{\circ}\left(0,67^{\circ}\right)$

$60 \gg 0,59^{\circ}\left(0,66^{\circ}\right)$

$75>0,45^{\circ}\left(0,52^{\circ}\right)$

$90 \times 0,30^{\circ}\left(0,37^{\circ}\right)$

$105 \gg 0,28^{\circ}\left(0,35^{\circ}\right)$

$120>0,28^{\circ}\left(0,35^{\circ}\right)$

$135 \gg 0,25^{\circ}\left(0,32^{\circ}\right)$

$150 \gg 0,16^{\circ}\left(0,23^{\circ}\right)$

165 ॰ $0,10^{\circ}\left(0,17^{\circ}\right)$

Wie die folgenden Versuche zeigen, ist es uns nicht geglückt, den Darmsaft und den Hefepreßsaft in ihrer Wirksamkeit sich völlig gleichzustellen. Es kann keinem Zweifel unterliegen, daß eine völlig scharfe Einstellung möglich ist. Da wir von d-Alanylglycin einen nur beschränkten Vorrat hatten und viele Vorversuche notwendig gewesen waren, mußten wir uns vorläufig mit einer annähernden Gleichstellung begnügen.

Versuch 3.

$1,0 \mathrm{ccm}$ d-Alanyl-glycinlösung

$(1 / 1000-\mathrm{Mol}$.).

1,0 . Hefepreßsaft (nach erfolgter Verdünnung mit Wasser).

4,5 \ Wasser.

Zeit Abgelesener Winkel

0 Minuten

20 .

55 จ

$1,00^{\circ}$

85 ,

$0,76^{\circ}$

$0,70^{\circ}$

$0,65^{\circ}$

115 .

$0,69^{\circ}$

145 จ

$0,69^{\circ}$

175

$6^{1 / 2}$ Stunden

$0,64^{\circ}$

$81 / 2$.

$10^{1 / 2}$,
Versuch 4 .

1,0 ccm d-Alanyl-glycinlösung

(1/1000-Mol.).

1,0 > Darmsaft.

4,5 Wasser.

Zeit Abgelesener Winkel

0 Minuten $1,00^{\circ}\left(1,15^{\circ}\right)$

$30 \quad 0,75^{\circ}\left(0,90^{\circ}\right)$

$65>0,66^{\circ}\left(0,81^{\circ}\right)$

$95>0,64^{\circ}\left(0,79^{\circ}\right)$

$125>0,62^{\circ}\left(0,77^{\circ}\right)$

$165 \Rightarrow 0,60^{\circ}\left(0,75^{\circ}\right)$

$335>0,32^{\circ}\left(0,47^{\circ}\right)$

$71 / 2$ Stunden $0,25^{\circ} \quad\left(0,40^{\circ}\right)$

$88^{3 / 4}>0,15^{0}\left(0,30^{\circ}\right)$

$91 / 2>0,12^{\circ}\left(0,27^{\circ}\right)$

1) Die eingeklammerten Zahlen geben das Drehungsvermögen unter Berücksichtigung der Eigendrehung der Fermentlösung wieder. 
Waren nun die im Darmsaft und im Hefepreßsaft enthaltenen peptolytischen Fermente verschiedenartig, so war zu erwarten, daß sie sich gegenüber verschiedenen optisch-aktiven Dipeptiden verschieden verhalten würden. Wir haben vorläufig das Verhalten der beiden, auf einander eingestellten Fermentlösungen auf Glycyl-l-leucin untersucht. Die folgende Übersicht gibt die erhaltenen Resultate wieder:

Versuch 5.

$1,00 \mathrm{ccm}$ Glycyl-1-leucinlösung (1/2000-Mol.).

$1,0 \gg$ Hefepreßsaft. $4,5 \gg$ Wasser.

\begin{tabular}{|c|c|c|}
\hline \multicolumn{2}{|c|}{ Zeit } & Abgelesener Winkel \\
\hline $0 \mathrm{M}$ & Iinuten & $-0,55^{\circ}$ \\
\hline 45 & $>$ & $-0,55^{0}$ \\
\hline 75 & > & $-0,53^{\circ}$ \\
\hline 150 & $\triangleright$ & $-0, \tilde{5} 3^{\circ}$ \\
\hline 270 & $\triangleright$ & $-0,50^{\circ}$ \\
\hline $71 / 2$ & Stunden & $-0,50^{\circ}$ \\
\hline 9 & $\triangleright$ & $-0,46^{\circ}$ \\
\hline $91 / 2$ & > & $-0,42^{\circ}$ \\
\hline $23^{3} / 4$ & $\triangleright$ & $-0,20^{\circ}$ \\
\hline $26^{3} / 4$ & $\triangleright$ & $-0,18^{\circ}$ \\
\hline $32^{3} / 4$ & $"$ & $-0,20^{\circ}$ \\
\hline 48 & $\triangleright$ & $-0,04^{0}$ \\
\hline
\end{tabular}

Versuch 6.

$1,0 \mathrm{ccm}$ Glycyl-l-leucinlösung ( $1 / 2000-\mathrm{Mol}$.).

1,0 ¿ Darmsaft.

$4, \tilde{0}$ \Wasser.

Zeit Abgelesener Winkel

0 Minuten $-0,55^{\circ}$

$30 \gg-0,50^{\circ}$

60 * $-0,50^{\circ}$

$135 \times-0,50^{\circ}$

270 > $-0,40^{\circ}$

$7^{1 / 4}$ Stunden $-0,36^{\circ}$

$8^{1 / 4}>\quad-0,31^{\circ}$

$8^{3} / 4>-0,30^{\circ}$

$23^{1 / 4}>-0,15^{\circ}$

$27 \quad-0,13^{\circ}$

$33 \quad-0,15^{\circ}$

$48^{1 / 2}> \pm 0^{\circ}$

Aus diesen Versuchen geht hervor, daß der Abbau des Glycyl-l-leucins bei beiden Fermentlösungen annähernd gleich rasch verlaufen ist. Diese Versuche müssen selbstverständlich auf ein viel reichhaltigeres Material von optisch-aktiven Dipeptiden ausgedehnt werden. Erst dann wird man zu sicheren Schlüssen gelangen. Diese Versuche sollen vorläufig nur den Weg andeuten, den wir zur Entscheidung des gestellten Problems eingeschlagen haben.

Eine sehr weitgehende Differenzierung der Wirkung verschiedener Fermentlösungen mußte sich durch die Verwendung von komplizierter gebauten optisch-aktiven Polypeptiden ermöglichen lassen. Wählen wir als Beispiel das Tripeptid d-Alanylglycyl-glycin. Es zeigt in wässeriger Lösung $[\alpha]_{20^{0}}^{\mathrm{D}}=+30^{\circ}$. Sein Abbau kann nun in der Weise erfolgen, daß zunächst 
hauptsächlich d-Alanin abgespalten wird. Es bleibt das Dipeptid Glycyl-glycin übrig. In diesem Falle muß das Drehungsvermögen der Lösung beständig abnehmen, denn Glycyl-glycin ist optisch inaktiv. Findet dagegen eine Abspaltung von Glycin statt, dann muß sich dieser Verlauf der Hydrolyse an einem Ansteigen des Drehungsvermögens kund geben, denn das entstehende Dipeptid d-Alanyl-glycin besitzt $[\alpha]_{20^{\circ}}^{\mathrm{D}}=+50^{\circ}$. Verwenden wir die zu vergleichenden, auf ein bestimmtes Dipeptid eingestellten Fermentlösungen, so können wir durch Benützung derartiger Tripeptide prüfen, ob der Abbau in genau der gleichen Weise verläuft oder nicht.

Wir haben einstweilen Hefepreßsaft und Darmsaft, die beide auf d-Alanyl-glycin eingestellt waren, auf d-Alanyl-glycylglycin und Glycyl-d-alanyl-glycin ${ }^{1}$ ) einwirken lassen. Die folgenden Schema erleichtern den Einblick in den Gang der Hydrolyse :

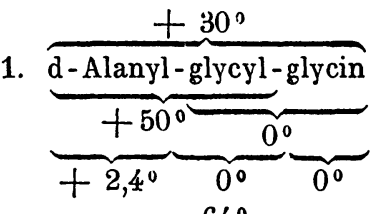

2. $\overbrace{\text { Glycyl-d-alanyl-glycin }}$

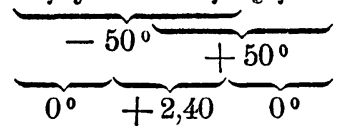

Versuch 7 .

$1,00 \mathrm{ccm}$ d-Alanyl-glycyl-glycinlösung (1/2000-Mol.).

1,0 Hefepreßsaft.

$4,5 \gg$ Wasser.

$\begin{array}{rr}\text { Zeit } & \text { Abgelesener W } \\ 0 \text { Minuten } & +0,53^{\circ} \\ 30 & +0,36^{\circ} \\ 60 & +0,31^{\circ} \\ 90 & +0,17^{\circ} \\ 210 & \\ 240 & \pm 0,10^{\circ} \\ 270 & \pm 0^{\circ} \\ 390 & \pm 0^{\circ}\end{array}$

Versuch 8.

$1,0 \mathrm{ccm}$ d-Alanyl-glycyl-glycinlösung ( $1 / 2000-\mathrm{Mol}$.).

1,0 Darmsaft.

4,5 \Wasser.

Zeit Abgelesener Winkel

$\begin{array}{rll}0 \text { Minuten } & +0,48^{\circ} \\ 30 & \searrow & +0,35^{\circ} \\ 60 & > & +0,24^{\circ} \\ 90 & > & +0,16^{\circ} \\ 210 & > & \pm 0,03^{\circ} \\ 240 & \searrow & \pm 0^{\circ} \\ 270 & > & \pm 0^{\circ} \\ 390 & \searrow & \pm 0^{\circ}\end{array}$

1) Vgl. bierzu : Emil Abderhalden und A. H. Koelker, Weiterer 
Versuch 9.

1,0 ccm Glycyl-d-alanyl-glycinlösung ( $1 / 2000-M o l$.$) .$

1,0 , Hefepreßsaft.

4,5 , Wasser.

Zeit Abgelesener Winkel

0 Minuten $-0,94^{\circ}$

30 . $-0,87^{\circ}$

$60 \therefore-0,70^{\circ}$

$90 \quad-0,60^{\circ}$

$120, \quad-0,40^{\circ}$

$150 \gg-0,30^{\circ}$

$180 \gg-0,18^{\circ}$

$210 \gg-0,13^{\circ}$

$240 .-0,07^{\circ}$

$270 \therefore-0,02^{\circ}$

$315 .+0,10^{\circ}$

$330>+0,10^{\circ}$

$18^{1 / 2}$ Stunden $+0,22^{\circ}$

$19>+0,20^{\circ}$

$19^{1 / 2}>\quad+0,25^{\circ}$

$20 \cdots+0,25^{\circ}$

$20^{1} / 2,+0,25^{\circ}$

$24^{1 / 2},+0,25^{\circ}$

43

$+0,12^{\circ}$
Versuch 10

$1 \mathrm{ccm}$ Glycyl-d-alanyl-glycinlösung ( $1 / 2000$-Mol.).

$1 \gg$ Darmsaft.

4,5 , Wasser.

Zeit Abgelesener Winkel

0 Minuten $-0,94^{\circ}\left(-0,79^{\circ}\right)$

$30>-0,36^{\circ}\left(-0,21^{\circ}\right)$

$50 \gg-0,20^{\circ}\left(-0,05^{\circ}\right)$

$65>-0,10^{\circ}\left(+0,05^{\circ}\right)$

$95:+0,08^{\circ}$

$125 .+0,16^{\circ}$

$155 \gg+0,25^{\circ}$

$185 \gg+0,31^{\circ}$

$215>+0,28^{\circ}$

$245: \quad+0,25^{\circ}$

$275 \otimes+0,25^{\circ}$

$305 \gg+0,25^{\circ}$

18 Stunden $-0,04^{\circ}$

$18^{1 / 2} \cdot \pm 0^{0}$

$19 \div \pm 0^{\circ}$

$20 \gg \pm 0^{\circ}$

$24> \pm 0^{\circ}$

$45^{1 / 4} \cdot \pm 0^{0}$

Aus diesen Versuchen geht ohne weiteres hervor, daß beide Fermentlösungen die beiden Tripeptide in genau der gleichen Weise abgebaut haben. In beiden Fällen wurde das d-Alanyl-glycyl-glycin zunächst in d-Alanin und Glycyl-glycin gespalten und das Glycyl-d-alanyl-glycin in Glykokoll und dAlanyl-glycin.

Es ist somit nach den bisherigen Versuchen nicht gelungen, Unterschiede im Verhalten der peptolytischen Fermente des Darmsaftes und des Hefepreßsaftes aufzufinden. Sie scheinen im Gegenteil identisch zu sein. Wir werden diese Versuche auf weitere Fermentlösungen und Polypeptide ausdehnen. Jedenfalls besitzen wir für keine anderen Fermentarten Methoden, die in so scharfer Weise gestatten, die entsprechenden Fermente verschiedener Herkunft auf ihre Identität $\mathrm{zu}$ prüfen.

Beitrag zur Kenntnis des Verlaufs der fermentativen Polypeptidspaltung unter verschiedenen Bedingungen, Diese Zeitschrift, Bd. LIV, S. 363, 1908. 
Über den Verlauf der fermentativen Polypeptidspaltung. VI. 347

Anhangsweise sei noch erwähnt, daß wir Preßsaft aus Aspergillus niger auf d-Alanyl-glycin einwirken ließen. Er spaltete dieses Dipeptid.

\begin{tabular}{|c|c|c|}
\hline \multicolumn{3}{|c|}{$1,0 \mathrm{ccm}$ d-Alanyl-glycinlösung $(1 / 1000-\mathrm{M}$} \\
\hline $1,0>$ & Pilzsaft. & \\
\hline 4,5 & Nasser. & \\
\hline & & Abgelesener Winkel \\
\hline $0 \mathrm{~s}$ & unde & $-1,14^{\circ}$ \\
\hline 1 & , & $-1,08^{\circ}$ \\
\hline $2 \mathrm{~S}$ & unden & $-1,07^{\circ}$ \\
\hline 3 & , & $-1,07^{\circ}$ \\
\hline 15 & D & $-0,75^{\circ}$ \\
\hline 16 & D & $-0,69^{\circ}$ \\
\hline 17 & > & $-0,65^{\circ}$ \\
\hline 18 & , & $-0,60^{\circ}$ \\
\hline 19 & , & $-0,60^{\circ}$ \\
\hline 21,5 & , & $-0,50^{\circ}$ \\
\hline 23 & $\triangleright$ & $-0,50^{\circ}$ \\
\hline 25 & , & $-0,40^{\circ}$ \\
\hline 37 & , & $-0,18^{\circ}$ \\
\hline 40 & 2 & $-0,14^{\circ}$ \\
\hline 44 & , & $-0,12^{\circ}$ \\
\hline
\end{tabular}

Aus Blättern von Drosera rotundifolia bereiteter Preßsaft griff Glycyl-l-tyrosin nicht an:

$1,0 \mathrm{ccm}$ Glycyl-1-tyrosinlösung (1/1000-Mol.).

1,0 , Droserapreßsaft.

4,5 , Wasser.

$\begin{array}{cc}\text { Zeit } & \text { Abgelesener Winkel } \\ 0 \text { Minuten } & +0,75^{\circ} \\ 30 & +0,75^{\circ} \\ 60 & +0,75^{\circ} \\ 50 \text { Stunden } & +0,75^{\circ} \\ 23^{1 / 2} & +0,75^{\circ}\end{array}$

